\title{
La difusión del conocimiento y los repositorios institucionales: la experiencia del Instituto Mexicano de Tecnología del Agua
}

Knowledge dissemination and institutional repositories: the experience of the Mexican Institute of Water Technology

\section{Verónica VARgas SuÁrez, Patricia NaVARro SuÁsteguI}

Instituto Mexicano de Tecnología del Agua, Paseo Cuauhnáhuac 8532, Progreso, 62550, Jiutepec, Morelos (México), veronica_vargas@tlaloc.imta.mx, pnavarro@tlaloc.imta.mx

\begin{abstract}
Resumen
Se exponen los principales retos que se han tenido que sortear para alcanzar la consolidación del repositorio del Instituto Mexicano de Tecnología del Agua. Entre los desafíos más importantes se encuentran, por un lado, las contraposiciones legales, que han despertado incertidumbre entre los investigadores, $y$, por el otro, la falta de una política institucional resuelta a crear una concienciación sobre la utilidad, para investigadores y la propia institución, de contar con un repositorio institucional.
\end{abstract}

Palabras clave: Repositorios institucionales. Legislación. Acceso abierto. Instituciones gubernamentales. Ciencias de la Tierra. Instituto Mexicano de Tecnología del Agua. México.

\section{Introducción}

Con el desarrollo de las TIC, el acceso a la información científica y académica se ha convertido en un bien público, siendo el repositorio institucional uno de los instrumentos más idóneos a emplear, ya que permite la organización, preservación y difusión de la información.

Paralelamente a este desarrollo tecnológico, ha surgido un conjunto de políticas, normativas y herramientas administrativas $y$ legales que constituyen el universo de mecanismos con los que las instituciones cuentan para promover el crecimiento y la consolidación de sus repositorios. Al mismo tiempo, estos mecanismos procuran, por un lado, ejercer el derecho de autor $y$, por el otro, el equilibrio entre los creadores, las instituciones que los apoyan y financian, y la sociedad que tiene el derecho al conocimiento y a beneficiarse de los resultados de las investigaciones científicas.

En México, a mediados de 2014 se adicionó a la Ley de Ciencia y Tecnología el capítulo relacionado con el acceso abierto a la información científica y el repositorio nacional (1), otorgándole al Consejo Nacional de Ciencia y Tecnolo-

\begin{abstract}
The main challenges that have had to be overcome in order to try to reach the consolidation of the Mexican Institute of Water Technology repository are exposed. Among the most important tasks are, on the one hand, the legal contrapositions, which have aroused uncertainty among researchers, and, on the other hand, the lack of a determined institutional policy to create awareness among researchers and the institution per se of the usefulness of having an institutional repository.
\end{abstract}

Keywords: Institutional repositories. Legislation. Open access. Government institutions. Earth Sciences. Mexican Institute of Water Technology. Mexico.

gía (Conacyt) la responsabilidad de diseñar e impulsar una estrategia nacional para democratizar la información científica en el país.

La producción científica considerada corresponde a trabajos de investigadores, tecnólogos, académicos y estudiantes de maestría, doctorado y posdoctorado, que sean resultado de la realización de actividades de investigación financiadas con recursos públicos o que se haya utilizado infraestructura pública en su realización.

El Instituto Mexicano de Tecnología del Agua (IMTA), como centro público de investigación, tiene el compromiso de dar a conocer el conocimiento científico que genera y desde hace cinco años cuenta con su repositorio institucional. El desarrollo de dicho repositorio no ha sido una tarea sencilla, pues a lo largo del camino se han tenido que superar diversidad de retos, como las contraposiciones entre las mismas políticas públicas aunadas a las normativas institucionales. A través de este proyecto se ha podido constatar que aquellas decisiones y acciones gubernamentales que definen las problemáticas públicas específicas a solucionar y los mecanismos particulares a utilizar para ello, no siempre van de la mano. 


\section{Metodología}

Se llevó a cabo el análisis de la problemática, en materia de derechos de autor y políticas institucionales, que se ha presentado durante la consolidación del repositorio institucional del IMTA. Se realizaron entrevistas a un grupo de investigadores y se revisaron las políticas internas para identificar procedimientos que aseguren la incorporación de la producción intelectual de los investigadores en el repositorio institucional.

A través de las entrevistas, pudimos percatarnos que el factor común es el escaso reconocimiento de la importancia de compartir los conocimientos generados y la inseguridad que sienten en relación con sus derechos de autor. Por su parte, la revisión de las políticas institucionales nos dejó claro que no existen instrumentos normativos que demanden la incorporación de los recursos generados.

Con el fin de identificar casos de repositorios en los cuales se presenten conflictos vinculados a contraposiciones legales, realizamos una búsqueda bibliográfica en diversas bases de datos, no encontrando un solo caso que mencione de manera directa esta situación. Sin embargo, encontramos algunos artículos que se refieren a aspectos legales en el manejo de derechos de autor.

Josep Vives (2005, p. 269) presenta un trabajo relacionado con los repositorios institucionales en España. Vives proporciona una serie de recomendaciones vinculadas con el derecho de autor y reconoce que éste es uno de los derechos mejor protegidos. Menciona el rol de la Ley de Propiedad Intelectual, sus ordenanzas y excepciones. Sin embargo, no hace referencia a contraposiciones con otras leyes.

Por su parte, Babini, González, López y Medici (2010) presentan el caso del repositorio institucional del Consejo Latinoamericano de Ciencias Sociales (CLACSO). En este estudio se señala que, dado lo extremadamente restrictivas de sus leyes, se optó porque cada centro de investigación de CLACSO definiera qué materiales podían ser difundidos en acceso abierto. Desde nuestro punto de vista, se optó por la salida más sencilla: políticas de información para cada centro evitando la difícil vía del consenso.

Sanllorenti, Pelaya y Willman (2011), mencionan los procedimientos seguidos para la obtención de permisos por parte del autor. Sin embargo, reconocen la falta de legislaciones nacionales que impulsen y respalden el avance del acceso abierto.

\section{El repositorio del IMTA}

EI IMTA es un organismo público descentralizado, creado en 1986, teniendo como uno de sus objetivos principales el contribuir a la gestión sostenible del agua a través del conocimiento, la tecnología, la formación de recursos humanos y la innovación. A partir de 2009 se integra al sistema de centros públicos de investigación del Conacyt y, como tal, adquiere el compromiso de compartir el conocimiento científico que genera con fondos públicos. Razón por la cual, y con el fin de contar con mecanismos que permitan identificar, organizar, preservar y difundir la producción intelectual del Instituto, el Centro de Conocimiento del Agua (Cenca), centro de información del IMTA, desarrolla la versión preliminar de su repositorio institucional durante 2011.

En 2012 se lleva a cabo formalmente la primera etapa, la cual consistió en la selección tanto del software libre como del material documental a incluir, diseño de la interfaz y la digitalización del material que lo requería. El repositorio inició con 29 libros editados por el IMTA y 173 artículos escritos por especialistas del propio Instituto y publicados en las revistas Ingeniería Hidráulica en México y Tecnología y Ciencias del Agua, ambas editadas por el IMTA.

La segunda etapa se realizó en 2013, periodo dedicado principalmente al mantenimiento y enriquecimiento de las colecciones del repositorio. En esta etapa se rediseñó la interfaz y se integraron las tesis de maestría y doctorado de los estudiantes egresados del programa de posgrado del IMTA. En 2014 se reorganizaron las comunidades y colecciones, y a partir de 2015 se incluyeron algunos informes de los proyectos desarrollados en el Instituto. Desde 2016 se ha estado trabajando en la propuesta para la consolidación del repositorio.

Cabe mencionar que éste está desarrollado con el software DSpace y para la organización de la información se ha considerado el modelo de metadatos Dublin Core. En cuanto a los términos que se emplean para la recuperación de los documentos, se ha desarrollado una lista de autoridades propia, basada en el tesauro de ingeniería sanitaria y ambiental de la Organización Panamericana de la Salud.

Actualmente el repositorio se puede consultar en http://repositorio.imta.mx. Está organizado en ocho comunidades y cuenta con 670 documentos: 120 libros, 201 artículos publicados en las revistas ya mencionadas, 49 tesis de maestría, 9 de doctorado y 291 informes de proyectos. Cabe aclarar que por el momento se ha suspendido la inclusión de más documentos; una 
vez que se actualice la versión del programa $D S p a c e$, se reiniciará con esta actividad.

Desde un principio, los objetivos del repositorio han sido, por un lado, dar a conocer a los ciudadanos en general qué se está haciendo con fondos públicos, en términos de investigación sobre los recursos hídricos del país; por el otro, crear un círculo virtuoso que permita a investigadores, que están trabajando la misma línea de investigación, colaborar mutuamente y así evitar la duplicidad de esfuerzos y un consumo innecesario de recursos humanos, financieros y materiales.

Aunado a lo dicho anteriormente, la intención de consolidar el repositorio institucional del IMTA ha sido cubrir, de alguna manera, los vacíos de información relacionada con el agua en México; vacíos provocados, principalmente, por la dificultad de acceder a la información generada en proyectos de investigación. De igual manera, se busca contribuir en la preservación del conocimiento generado en el Instituto e impulsar su visibilidad en la comunidad a la que se debe.

Dada la complejidad de los aspectos legales de propiedad intelectual, el repositorio sólo incluye la producción científica generada por el propio IMTA y por investigadores del Instituto que han publicado en las revistas ya mencionadas. Desde la creación del repositorio, todos los documentos han estado protegidos por la licencia Creative Commons Atribución-No Comercial 2.5 México.

\section{La consolidación}

Dentro de la consolidación del repositorio del IMTA se han tenido que considerar aspectos tanto técnicos como legales. Los primeros se refieren, principalmente, a la actualización del software libre y a la adecuación de los registros de acuerdo a los lineamientos del Conacyt, para conseguir la interoperabilidad con el Repositorio Nacional. Los segundos, se han identificado como una de las principales dificultades para el fortalecimiento del repositorio. En este sentido, vale mencionar que, dentro de los lineamientos establecidos por el Conacyt para ser cosechado en el Repositorio Nacional, es necesaria la obtención de cartas de autorización por parte de los autores, por cada obra incluida en el repositorio; es de resaltar que esta labor ha sido una de las actividades más complicadas.

Las contraposiciones en algunas disposiciones legislativas han provocado desconfianza por parte de los autores y se refleja en su negativa a firmar dicha carta, misma que ha sido redactada por el área jurídica del IMTA y se basa en el artículo 83 de la Ley Federal del Derecho de Autor, la cual establece, grosso modo, que el IMTA tiene la facultad para la divulgación de las obras generadas por sus investigadores, a los cuales ha remunerado. Situación que hace inferir que las cartas de autorización podrían omitirse, ya que el repositorio compila únicamente libros producidos por el propio IMTA, artículos publicados en las revistas del Instituto, informes de proyectos de investigaciones realizadas por el IMTA y financiadas con recursos públicos, y tesis de maestría y doctorado de los estudiantes egresados de los programas de posgrado del IMTA.

Por su parte, la Ley de Ciencia y Tecnología (Mexico, 2015) establece que las investigaciones financiadas con recursos públicos o que hayan utilizado infraestructura pública en su realización deben ser de acceso abierto. Sin embargo, la misma ley atribuye al autor la decisión personal de que su investigación pueda ser confidencial o reservada (artículos 65 y 69) y, por ende, no ser de acceso abierto.

Al ser una decisión personal y no un mandato, se corre el riesgo de no poder concentrar toda la producción intelectual del IMTA, razón de ser de su repositorio. Ya lo mencionan estudiosos como Carr, Swan y Harnad (2011), quienes resaltan el hecho de que la mayoría de los autores no comparten sus investigaciones, a menos de que tengan el mandato explícito de las instituciones que los han financiado. En el mejor de los casos, esta práctica puede llegar a ser una buena costumbre después de algunos años.

Por otro lado, el desconocimiento por parte de los investigadores del IMTA de la existencia del ORCID (Open Researcher and Contributor ID), identificador obligado en los registros cosechables por el Conacyt, ha representado una barrera más en la consolidación del repositorio. A pesar de que el Cenca puede crear cuentas para los investigadores del IMTA, no ha tomado esta iniciativa ya que la gestión de las mismas está restringida a los propios investigadores, quienes tendrían que reclamar dichas cuentas y proceder a registrar en ellas sus publicaciones $y$, como es de suponer, el Cenca no puede garantizar que los investigadores lo lleven a cabo.

De igual manera, no se pueden ignorar las normativas institucionales a nivel federal, pues si bien existe una Estrategia Digital Nacional, a través de la cual el Estado pretende garantizar a la ciudadanía el derecho de acceso a las TIC y la seguridad de la información, la realidad ha mostrado que la complejidad de los trámites burocráticos retrasa sobremanera y en ocasiones impide alcanzar los objetivos del proyecto. 
Baste mencionar, como ejemplo, el tiempo que tomó contar con la autorización gubernamental para la contratación del servicio de actualización del software libre DSpace, ya que el Cenca no cuenta con el personal calificado para esta tarea.

Por último, pero no por ello menos importante, es preciso mencionar el desconocimiento de la existencia del repositorio institucional por una buena parte de los investigadores del IMTA. Situación que se ha hecho más evidente con la petición de las firmas de autorización. Vale resaltar que, desde su creación, el repositorio ha estado alojado dentro del sitio del Cenca (http://cenca.imta.mx). En 2013, a través del trabajo presentado en el congreso anual del IMTA, El repositorio como instrumento que multiplica la visibilidad institucional, se mostró formalmente al Instituto. Además, en los cursos que el personal del Cenca imparte anualmente sobre desarrollo de habilidades informativas, se explica cómo opera el repositorio.

\section{Conclusiones}

Los retos a afrontar para consolidar de una manera contundente el repositorio del IMTA, son, por un lado, construir políticas institucionales que conviertan en mandato el compartir los resultados de las investigaciones financiadas con recursos e infraestructura públicos. Por el otro, traspasar institucionalmente la noción de "transferir conocimiento" para llegar al concepto de "compartir conocimiento", para lo cual será necesario realizar un mayor esfuerzo de comunicación y convencimiento para promover entre los investigadores del IMTA el registro en el ORCID y en la firma de las cartas de autorización.

Por consiguiente, es necesario un planteamiento que considere una planeación estratégica muy específica, donde las metas y los tiempos sean concretos y el avance paulatino, antes de que sea un esfuerzo más en vacío.

A nuestro parecer, independientemente de las contraposiciones de la legislación en México, los investigadores deberían pensar más allá de las regalías, es decir, en la posibilidad de un reconocimiento y la divulgación de su obra; y las instituciones, deberían dirigir sus esfuerzos para facilitar el acceso al conocimiento generado con recursos públicos.

Si bien es cierto que, por el momento, la inclusión de obras en el repositorio depende del consentimiento de los autores, el crecimiento de éste no puede depender únicamente de su voluntad. Se tendrá que considerar la realización de una campaña de promoción significativa, que se apoye en una política institucional comprometida con el Repositorio Nacional. Un ejercicio esencial para impulsar dicha política es la realización de campañas de promoción que permitan el conocimiento a investigadores y población en general sobre los trabajos que lleva a cabo el IMTA e invite a la participación y uso del repositorio institucional, para posibilitar un mayor acceso a la información y así colaborar en la democratización de la información científica en el país.

\section{Notas}

(1) Definición de repositorio que da la Ley de Ciencia y Tecnología (2014): "Repositorio, la plataforma digital centralizada que, siguiendo estándares internacionales, almacena, mantiene y preserva la información científica, tecnológica y de innovación, la cual se deriva de las investigaciones, productos educativos y académicos" (artículo 4, fracción XII)

\section{Referencias}

Babini, Dominique; González, Jessica, López, Fernando; Medici, Flavia (2010). Construcción social de repositorios institucionales: el caso de un repositorio de América Latina y el Caribe. // Información, cultura y sociedad: revista del Instituto de Investigaciones Bibliotecológicas. 23, 63-90

Vives i Gràcia, Josep (2005). Aspectos de propiedad intelectual en la creación y gestión de repositorios institucionales. // El profesional de la información. 14:1, 267-278

Carr, Les; Swan, Alma; Harnad, Stevan (2011). Creating and Curating the Cognitive Commons: Southampton's Contribution. // Simons, Maarten; Decuypere, Mathias; Vlieghe, Joris; Masschelein, Jan (eds.) Curating the European University. Leuven: Leuven University Press, 2011. 193-199.

Consejo Nacional de Ciencia y Tecnología [2014]. Lineamientos técnicos para el Repositorio Nacional y los repositorios institucionales. http://www.siicyt.gob.mx/index.php/ normatividad/2-conacyt/4-conacyt/1499-lineamientos-tec nicos-para-el-repostitorio-nacional-y-los-reporsitorios-ins titucionales/file (2017-03-06).

México (2015). Ley de Ciencia y Tecnología. Última reforma publicada DOF 08-12-2015. http://www.diputados.gob. mx/LeyesBiblio/pdf/242_081215.pdf (2017-03-06).

México (2016). Ley Federal del Derecho de Autor. Última reforma publicada DOF 13-01-2016. http://www.diputado s.gob.mx/LeyesBiblio/pdf/122_130116.pdf (2017-03-06).

Sanllorenti, Ana María; Pelaya, Lucía; Willman, Martín (2011). Instrumentos de gestión del derecho de autor en repositórios de acceso abierto. // Revista Interamericana de Bibliotecología. 34:3, 313-328.

Enviado: 2017-03-27. Segunda versión: 2017-05-25. Aceptado: 2017-09-12. 